\title{
Eigenvalue Bounds for Schrödinger Operators with a Homogeneous Magnetic Field
}

\author{
RUPERT L. FRANK ${ }^{1}$ and RIKARD OLOFSSON ${ }^{2}$ \\ ${ }^{1}$ Department of Mathematics, Princeton University, Princeton, NJ 08544, USA. \\ e-mail: rlfrank@math.princeton.edu \\ ${ }^{2}$ Department of Mathematics, Uppsala University, Box 480, 75106 Uppsala, Sweden. \\ e-mail: rikard.olofsson@math.uu.se
}

Received: 13 January 2011 / Revised: 10 May 2011 / Accepted: 10 May 2011

Published online: 9 June 2011 - (C) The Author(s) 2011

\begin{abstract}
We prove Lieb-Thirring inequalities for Schrödinger operators with a homogeneous magnetic field in two and three space dimensions. The inequalities bound sums of eigenvalues by a semi-classical approximation which depends on the strength of the magnetic field, and hence quantifies the diamagnetic behavior of the system. For a harmonic oscillator in a homogenous magnetic field, we obtain the sharp constants in the inequalities.
\end{abstract}

Mathematics Subject Classification (2000). Primary 35P15; Secondary 35J10, 81Q10.

Keywords. Schrödinger operator, Lieb-Thirring inequalities, magnetic field.

\section{Introduction and Main Result}

Lieb-Thirring inequalities [15] provide bounds on the sum of negative eigenvalues of Schrödinger operators in terms of a phase space integral. In this paper, we are interested in two-dimensional Schrödinger operators $H_{B}+V$ with a homogenous magnetic field of strength $B>0$. Here

$$
H_{B}=\left(-i \frac{\partial}{\partial x_{1}}+\frac{B x_{2}}{2}\right)^{2}+\left(-i \frac{\partial}{\partial x_{2}}-\frac{B x_{1}}{2}\right)^{2}
$$

is the Landau Hamiltonian in $L^{2}\left(\mathbb{R}^{2}\right)$ and $V$ is a real-valued function. The LiebThirring inequality states that

$$
\operatorname{Tr}\left(H_{B}+V\right)_{-} \leq r_{2}(2 \pi)^{-2} \iint_{\mathbb{R}^{2} \times \mathbb{R}^{2}}\left(|p|^{2}+V(x)\right)_{-} \mathrm{d} x \mathrm{~d} p
$$

(C) 2011 by the authors. This paper may be reproduced, in its entirety, for non-commercial purposes. 
with the (currently best, but presumably non-optimal) constant $r_{2}=\pi / \sqrt{3}$ from [3]. Physically, the left side is (minus) the energy of a system of non-interacting fermions in an external potential $V$ and an external, homogeneous magnetic field of strength $B$, whereas the right side is $-r_{2}$ times a semi-classical approximation to that energy.

Physically, one expects the system to show a diamagnetic behavior, that is, to have a higher energy in the presence of a magnetic field. This is however not reflected in (1), which has a right hand side independent of $B$. We refer to [6] for further references and a survey over this problem. Our goal in this letter is to obtain a bound similar to (1), but with a more refined semi-classical approximation which takes $B$ into account. The approximation we propose is:

$$
\frac{B}{2 \pi} \sum_{m=0}^{\infty} \int_{\mathbb{R}^{2}}((2 m+1) B+V(x))_{-} \mathrm{d} x .
$$

This quantity reflects the diamagnetic behavior since,

$$
\frac{B}{2 \pi} \sum_{m=0}^{\infty} \int_{\mathbb{R}^{2}}((2 m+1) B+V(x))_{-} \mathrm{d} x \leq \frac{1}{(2 \pi)^{2}} \iint_{\mathbb{R}^{2} \times \mathbb{R}^{2}}\left(|p|^{2}+V(x)\right)_{-} \mathrm{d} x \mathrm{~d} p
$$

for every $V$. Inequality (3) follows (even before the $x$-integration) from an easy convexity inequality (see Lemma 12 below). We also note that when $B \rightarrow 0$, by a Riemann sum argument, the quantity (2) approaches

$$
(4 \pi)^{-1} \int_{0}^{\infty} \mathrm{d} E \int_{\mathbb{R}^{2}}(E+V(x))_{-} \mathrm{d} x=(2 \pi)^{-2} \iint_{\mathbb{R}^{2} \times \mathbb{R}^{2}}\left(|p|^{2}+V(x)\right)_{-} \mathrm{d} x \mathrm{~d} p,
$$

which is the 'usual' phase space integral.

While the right side of (1) (up to the constant $r_{2}$ ) has the correct limiting behavior when a small parameter $\hbar$ is introduced, it is not useful in the coupled limit $B \rightarrow \infty$ and $\hbar \rightarrow 0$. This limit is physically relevant, for instance, in the study of neutron stars [13]. The magnetic quantity (2) reproduces the correct behavior in this regime. It is remarkable that this asymptotic profile is, indeed, a uniform, nonasymptotic bound. This is implicitly contained in [14] who use, however, only an approximation of (2). Our first result is:

THEOREM 1. For any $B>0$ and any $V$ on $\mathbb{R}^{2}$ one has,

$$
\operatorname{Tr}\left(H_{B}+V\right)_{-} \leq \rho_{2} \frac{B}{2 \pi} \sum_{m=0}^{\infty} \int_{\mathbb{R}^{2}}((2 m+1) B+V(x))_{-} \mathrm{d} x
$$

with $\rho_{2}=3$. 
Hence, up to the moderate increase from $r_{2}=\pi / \sqrt{3} \approx 1.81$ to $\rho_{2}=3$, we have found a magnetic analogue of (1) which reflects the desired diamagnetic behavior (3). An important ingredient in our proof is a method developed recently by Rumin [16] to derive kinetic energy inequalities; see Section 2.1.

Similarly as in the non-magnetic case, one might ask for the optimal value of the constant $\rho_{2}$. By the semi-classical result mentioned above one necessarily has $\rho_{2} \geq 1$. A first result in this direction was obtained in [7] (extending previous work of [4]), where it was shown that if one takes $V$ to be constant on a set of finite measure and plus infinity otherwise, then (5) holds with $\rho_{2}=1$. Our second main result is an analogous optimal bound for a harmonic oscillator.

THEOREM 2. For any $B>0, \omega_{1}>0, \omega_{2}>0$ and $\mu>0$, inequality (5) holds with $\rho_{2}=1$ for $V(x)=\omega_{1}^{2} x_{1}^{2}+\omega_{2}^{2} x_{2}^{2}-\mu$.

In particular, letting $B \rightarrow 0$ and using the limit in (4) we recover the known bounds in the non-magnetic case from [2,11]. Even though the eigenvalues of a harmonic oscillator in a homogeneous magnetic field are explicitly known (Lemma 10), the proof of Theorem 2 relies on a delicate property of a subclass of convex functions (Lemma 14) which, we feel, could be useful even beyond the context of this paper.

Moments of eigenvalues. Using some by now standard techniques we derive a few consequences of Theorems 1 and 2. First, following Aizenman and Lieb [1] one can replace $V$ by $V-\mu$ in (5) and integrate with respect to $\mu$ to obtain that for any $\gamma \geq 1$

$$
\operatorname{Tr}\left(H_{B}+V\right)_{-}^{\gamma} \leq \rho_{2} \frac{B}{2 \pi} \sum_{m=0}^{\infty} \int_{\mathbb{R}^{2}}((2 m+1) B+V(x))_{-}^{\gamma} \mathrm{d} x,
$$

where $\rho_{2}=3$ for general $V$ and $\rho_{2}=1$ for $V(x)=\omega_{1}^{2} x_{1}^{2}+\omega_{2}^{2} x_{2}^{2}-\mu$. The restriction $\gamma \geq 1$ is necessary, since one easily checks that for $0 \leq \gamma<1$ there is no constant $\rho_{2}$ such that (6) holds for all potentials $V$. Restricting ourselves to the quadratic case we shall show in Section 3.4

PROPOSITION 3. For any $0 \leq \gamma<1$ there are $B>0, \mu>0$ and $\omega_{1}=\omega_{2}$ such that for $V(x)=\omega_{1}^{2} x_{1}^{2}+\omega_{2}^{2} x_{2}^{2}-\mu$ one has,

$$
\operatorname{Tr}\left(H_{B}+V\right)_{-}^{\gamma}>\frac{B}{2 \pi} \sum_{m=0}^{\infty} \int_{\mathbb{R}^{2}}((2 m+1) B+V(x))_{-}^{\gamma} \mathrm{d} x .
$$

In particular, this shows that (6) does not hold with $\rho_{2}=1$ if $0 \leq \gamma<1$, not even for a quadratic potential. Our counterexample in Proposition 3 appears in the limit 
$\omega_{j} / B \rightarrow 0$ (with $\mu / B=3$ fixed). Another counterexample can be obtained in the $B \rightarrow 0$ limit from the (non-magnetic) counterexample of Helffer-Robert [8] and the fact that for potentials of the special form $V(x)=\omega_{1}^{2} x_{1}^{2}+\omega_{2}^{2} x_{2}^{2}-\mu$ one has

$$
\frac{B}{2 \pi} \sum_{m=0}^{\infty} \int_{\mathbb{R}^{2}}((2 m+1) B+V(x))_{-}^{\gamma} \mathrm{d} x \leq \frac{1}{(2 \pi)^{2}} \iint_{\mathbb{R}^{2} \times \mathbb{R}^{2}}\left(|p|^{2}+V(x)\right)_{-}^{\gamma} \mathrm{d} x \mathrm{~d} p
$$

for all $\gamma \geq 0$ [and not only for $\gamma \geq 1$, as in (3)].

Three dimensions. Next, we shall show that our bounds for $d=2$ can be applied to deduce analogous bounds for $d=3$. This argument is in the spirit of the lifting argument from [10-12]. We denote by $\hat{H}_{B}=H_{B}-\frac{\partial^{2}}{\partial x_{3}^{2}}$ the Landau Hamiltonian in $L^{2}\left(\mathbb{R}^{3}\right)$.

COROLLARY 4. For any $B>0$ and any $\hat{V}$ on $\mathbb{R}^{3}$, one has

$$
\operatorname{Tr}\left(\hat{H}_{B}+\hat{V}\right)_{-} \leq \rho_{3} \frac{B}{(2 \pi)^{2}} \sum_{m=0}^{\infty} \iint_{\mathbb{R}^{3} \times \mathbb{R}}\left((2 m+1) B+p_{3}^{2}+\hat{V}(x)\right)_{-} \mathrm{d} x \mathrm{~d} p_{3}
$$

with $\rho_{3}=\sqrt{3} \pi$.

Proof. From the operator-valued Lieb-Thirring inequality of [3] we know that

$$
\operatorname{Tr}\left(\hat{H}_{B}+\hat{V}\right)_{-} \leq \frac{\pi}{\sqrt{3}} \iint_{\mathbb{R} \times \mathbb{R}} \operatorname{Tr}_{L^{2}\left(\mathbb{R}^{2}\right)}\left(H_{B}+p_{3}^{2}+\hat{V}\left(\cdot, x_{3}\right)\right)_{-} \frac{\mathrm{d} x_{3} \mathrm{~d} p_{3}}{2 \pi} .
$$

Inequality (8) is therefore a consequence of Theorem 1.

For the harmonic oscillator we have:

COROLLARY 5. For any $B>0, \omega_{1}>0, \omega_{2}>0, \omega_{3}>0$ and $\mu>0$, inequality (8) holds with $\rho_{3}=1$ for $\hat{V}(x)=\omega_{1}^{2} x_{1}^{2}+\omega_{2}^{2} x_{2}^{2}+\omega_{3}^{2} x_{3}^{2}-\mu$.

Proof. We denote by $E_{j}$ the eigenvalues of the one-dimensional harmonic oscillator $H=-\frac{\partial^{2}}{\partial x_{3}^{2}}+\omega_{3}^{2} x_{3}^{2}$. Then, since $\hat{H}_{B}+\hat{V}=\left(H_{B}+V\right) \otimes I+I \otimes H$ with $V\left(x_{1}, x_{2}\right)=$ $\omega_{1}^{2} x_{1}^{2}+\omega_{2}^{2} x_{2}^{2}$, we have

$$
\operatorname{Tr}_{L^{2}\left(\mathbb{R}^{3}\right)}\left(\hat{H}_{B}+\hat{V}\right)_{-}=\sum_{j} \operatorname{Tr}_{L^{2}\left(\mathbb{R}^{2}\right)}\left(H_{B}+V+E_{j}-\mu\right)_{-}
$$


According to Theorem 2 (which trivially holds for $\mu \leq 0$ as well), this is bounded from above by

$$
\begin{aligned}
& \frac{B}{2 \pi} \sum_{j} \sum_{m=0}^{\infty} \int_{\mathbb{R}^{2}}\left((2 m+1) B+V\left(x_{1}, x_{2}\right)+E_{j}-\mu\right)_{-} \mathrm{d} x_{1} d x_{2} \\
& \quad=\frac{B}{2 \pi} \sum_{m=0}^{\infty} \int_{\mathbb{R}^{2}} \operatorname{Tr}_{L^{2}(\mathbb{R})}\left(H+(2 m+1) B+V\left(x_{1}, x_{2}\right)-\mu\right)_{-} \mathrm{d} x_{1} \mathrm{~d} x_{2} .
\end{aligned}
$$

Next, we shall use that $H$ satisfies a Lieb-Thirring inequality with semi-classical constant $[2,11]$, that is, for any $\Lambda \in \mathbb{R}$,

$$
\operatorname{Tr}_{L^{2}(\mathbb{R})}(H-\Lambda)_{-} \leq \frac{1}{2 \pi} \iint_{\mathbb{R} \times \mathbb{R}}\left(p_{3}^{2}+\omega_{3}^{2} x_{3}^{2}-\Lambda\right)_{-} \mathrm{d} x_{3} \mathrm{~d} p_{3}
$$

(This can also be seen from Lemma 12 and recalling the explicit form of the eigenvalues of $H$.) It follows that for every fixed $\left(x_{1}, x_{2}\right)$

$$
\begin{aligned}
& \operatorname{Tr}_{L^{2}(\mathbb{R})}\left(H+(2 m+1) B+V\left(x_{1}, x_{2}\right)-\mu\right)_{-} \\
& \quad \leq \frac{1}{2 \pi} \iint_{\mathbb{R} \times \mathbb{R}}\left(p_{3}^{2}+\omega_{3}^{2} x_{3}^{2}+(2 m+1) B+V\left(x_{1}, x_{2}\right)-\mu\right)_{-} \mathrm{d} x_{3} \mathrm{~d} p_{3},
\end{aligned}
$$

which proves the claimed bound.

Remark 6. The previous proof shows that (8) with $\rho_{3}=1$ is valid for more general potentials $\hat{V}(x)=V\left(x_{1}, x_{2}\right)+v\left(x_{3}\right)$, where $V\left(x_{1}, x_{2}\right)=\omega_{1}^{2} x_{1}^{2}+\omega_{2}^{2} x_{2}^{2}$ and where $v$ is such that $\operatorname{Tr}_{L^{2}(\mathbb{R})}\left(-\frac{\mathrm{d}^{2}}{\mathrm{~d} x_{3}^{2}}+v\left(x_{3}\right)-\Lambda\right)_{-} \leq \frac{1}{2 \pi} \iint_{\mathbb{R} \times \mathbb{R}}\left(p_{3}^{2}+v\left(x_{3}\right)-\Lambda\right)_{-} \mathrm{d} x_{3} \mathrm{~d} p_{3}$ for all $\Lambda$.

A similar argument as in the proofs of Corollaries 4 and 5 (based on the operator-valued Lieb-Thirring inequalities of $[9,12])$ shows that for general $\hat{V}$ one has

$$
\operatorname{Tr}\left(\hat{H}_{B}+\hat{V}\right)_{-}^{\gamma} \leq \rho_{3, \gamma} \frac{B}{(2 \pi)^{2}} \sum_{m=0}^{\infty} \iint_{\mathbb{R}^{3} \times \mathbb{R}}\left((2 m+1) B+p_{3}^{2}+\hat{V}(x)\right)_{-}^{\gamma} \mathrm{d} x \mathrm{~d} p_{3}
$$

with $\rho_{3, \gamma}=6$ if $\gamma \geq 1 / 2$, with $\rho_{3, \gamma}=\pi \sqrt{3}$ if $\gamma \geq 1$ and with $\rho_{3, \gamma}=3$ if $\gamma \geq 3 / 2$. Moreover, in the special case of $\hat{V}(x)=\omega_{1}^{2} x_{1}^{2}+\omega_{2}^{2} x_{2}^{2}+\omega_{3}^{2} x_{3}^{2}-\mu$, (9) holds with $\rho_{3}=1$ for $\gamma \geq 1$ and with $\rho_{3, \gamma}=2(\gamma /(\gamma+1))^{\gamma}$ for $0 \leq \gamma<1$. The latter follows from the fact [7] that

$$
\operatorname{Tr}_{L^{2}(\mathbb{R})}\left(-\frac{\mathrm{d}^{2}}{\mathrm{~d} x_{3}^{2}}+\omega_{3}^{2} x_{3}^{2}-\Lambda\right)_{-}^{\gamma} \leq 2\left(\frac{\gamma}{\gamma+1}\right)^{\gamma} \frac{1}{2 \pi} \iint_{\mathbb{R} \times \mathbb{R}}\left(p_{3}^{2}+\omega_{3}^{2} x_{3}^{2}-\Lambda\right)_{-}^{\gamma} \mathrm{d} x_{3} \mathrm{~d} p_{3} .
$$




\section{Proof of Theorem 1}

\subsection{A KINETIC ENERGY INEQUALITY}

We define a piecewise affine function $j:[0, \infty) \rightarrow[0, \infty)$ by

$$
j(\rho)=\frac{B^{2}}{2 \pi}\left(L^{2}+(2 L+1) r\right) \quad \text { if } \rho=\frac{B}{2 \pi}(L+r), L \in \mathbb{N}_{0}, r \in[0,1) .
$$

We note that $j$ is continuous, increasing and convex. One has $j(\rho)=B \rho$ if $\rho \leq$ $B /(2 \pi)$ and $j(\rho) \sim 2 \pi \rho^{2}$ if $\rho \gg B$. The connection between this function and the right side of (5) will become clearer in the next section.

THEOREM 7. Let $0 \leq \gamma \leq 1$ be a density matrix on $L^{2}\left(\mathbb{R}^{2}\right)$ with finite kinetic energy. Then

$$
\operatorname{Tr} H_{B} \gamma \geq 3 \int_{\mathbb{R}^{2}} j\left(\rho_{\gamma}(x) / 3\right) \mathrm{d} x,
$$

where $\rho_{\gamma}(x)=\gamma(x, x)$.

It is easy to see that $3 j(\rho / 3) \geq(1 / 3) j(\rho)$ for all $\rho \geq 0$, and therefore we also have

$$
\operatorname{Tr} H_{B} \gamma \geq(1 / 3) \int_{\mathbb{R}^{2}} j\left(\rho_{\gamma}(x)\right) \mathrm{d} x .
$$

Proof. The first part of our proof follows the method introduced by Rumin [16]. We define $j_{R}:[0, \infty) \rightarrow[0, \infty)$ by

$$
j_{R}(\rho)=B \rho+2 B \sum_{k=1}^{\infty}\left(\sqrt{\rho}-\sqrt{\frac{B k}{2 \pi}}\right)_{+}^{2} .
$$

We note that $j_{R}$ is differentiable and convex, $j_{R}(\rho)=B \rho$ if $\rho \leq B /(2 \pi)$ and $j_{R}(\rho) \sim$ $2 \pi \rho^{2} / 3$ if $\rho \gg B$. We shall first show that

$$
\operatorname{Tr} H_{B} \gamma \geq \int_{\mathbb{R}^{2}} j_{R}\left(\rho_{\gamma}(x)\right) \mathrm{d} x .
$$

In the second part of our proof (see Lemma 8) we show that $j_{R}(\rho) \geq 3 j(\rho / 3)$ for all $\rho \geq 0$.

For the proof of (10) we write:

$$
\operatorname{Tr} H_{B} \gamma=\int_{0}^{\infty} \operatorname{Tr}\left(P^{E} \gamma\right) \mathrm{d} E=\int_{\mathbb{R}^{2}} \int_{0}^{\infty} \rho_{\gamma}^{E}(x) \mathrm{d} E \mathrm{~d} x,
$$


where $P^{E}$ is the spectral projection of $H_{B}$ corresponding to the interval $[E, \infty)$ and where $\rho_{\gamma}^{E}(x)=\left(P^{E} \gamma P^{E}\right)(x, x)$. It is well known that,

$$
\left(1-P^{E}\right)(x, x)=\frac{B}{2 \pi} \#\left\{m \in \mathbb{N}_{0}:(2 m+1) B<E\right\} .
$$

The same clever use of the triangle inequality as in [16] leads to the pointwise lower bound:

$$
\rho_{\gamma}^{E}(x) \geq\left(\sqrt{\rho_{\gamma}(x)}-\sqrt{\frac{B}{2 \pi} \#\left\{m \in \mathbb{N}_{0}:(2 m+1) B<E\right\}}\right)_{+}^{2} .
$$

Indeed, if $\|\cdot\|_{2}$ denotes the Hilbert-Schmidt norm, we have for any set $\Omega$ of finite measure

$$
\begin{aligned}
\sqrt{\int_{\Omega} \rho_{\gamma}(x) \mathrm{d} x} & =\left\|\gamma^{1 / 2} \chi_{\Omega}\right\|_{2} \leq\left\|\gamma^{1 / 2} P_{E} \chi_{\Omega}\right\|_{2}+\left\|\gamma^{1 / 2}\left(1-P_{E}\right) \chi_{\Omega}\right\|_{2} \\
& \leq\left\|\gamma^{1 / 2} P_{E} \chi_{\Omega}\right\|_{2}+\left\|\left(1-P_{E}\right)^{1 / 2} \chi_{\Omega}\right\|_{2} \\
& =\sqrt{\int_{\Omega} \rho_{\gamma}^{E}(x) \mathrm{d} x}+\sqrt{|\Omega| \frac{B}{2 \pi} \#\left\{m \in \mathbb{N}_{0}:(2 m+1) B<E\right\}} .
\end{aligned}
$$

Choosing $\Omega$ to be a ball around any fixed point $x$ we obtain, as the radius of this ball tends to zero, that

$$
\sqrt{\rho_{\gamma}(x)} \leq \sqrt{\rho_{\gamma}^{E}(x)}+\sqrt{\frac{B}{2 \pi} \#\left\{m \in \mathbb{N}_{0}:(2 m+1) B<E\right\}} .
$$

This implies the claimed bound (12).

Inserting bound (12) in (11) we obtain

$$
\begin{aligned}
\operatorname{Tr} H_{B} \gamma & \geq \int_{\mathbb{R}^{2}}\left(\int_{0}^{B} \rho_{\gamma}(x) \mathrm{d} E+\sum_{k=1}^{\infty} \int_{(2 k-1) B}^{(2 k+1) B}\left(\sqrt{\rho_{\gamma}(x)}-\sqrt{\frac{B k}{2 \pi}}\right)_{+}^{2} \mathrm{~d} E\right) \mathrm{d} x \\
& =\int_{\mathbb{R}^{2}} j_{R}\left(\rho_{\gamma}(x)\right) \mathrm{d} x .
\end{aligned}
$$

This completes the proof of (10) and also, by Lemma 8 below, the proof of the theorem.

LEMMA 8. $j_{R}(\rho) \geq 3 j(\rho / 3)$ for all $\rho \geq 0$.

Proof. We are going to prove that

$$
j_{R}(3 \rho) \geq 3 j(\rho) .
$$


Note that this is an equality for $\rho \leq B /(6 \pi)$. Moreover, since the left side of (13) is convex and the right side linear for $\rho \leq B /(2 \pi)$, we conclude that (13) holds for all $\rho \leq B /(2 \pi)$.

Henceforth, we shall assume that $\rho \geq B /(2 \pi)$ and we write $3 \rho=(B / 2 \pi)(K+s)$ for some integer $K \geq 3$ and some $s \in[0,1)$. If $K=3 L+m$ with $L \in \mathbb{N}$ and $m \in$ $\{0,1,2\}$, then the lemma says that

$$
K+s+2 \sum_{k=1}^{K}(\sqrt{K+s}-\sqrt{k})^{2} \geq 3\left(L^{2}+\frac{1}{3}(2 L+1)(m+s)\right) .
$$

We expand the square on the left side and insert $L=(K-m) / 3$ on the right side. This shows that the assertion is equivalent to

$$
K+s+2 K(K+s)-4 \sqrt{K+s} \sum_{k=1}^{K} \sqrt{k}+K(K+1) \geq \frac{1}{3} K^{2}+\frac{2}{3} K s+s+R,
$$

for $K \in \mathbb{N}$ and $s \in[0,1)$, where $R=-\frac{1}{3} m^{2}-\frac{2}{3} m s+m$. Since the inequality has to be true for any $m \in\{0,1,2\}$, we can replace $R$ by its maximum over these $m$ (with fixed $s)$, that is, by $(2 / 3)(1-s)$. Thus, (13) is equivalent to

$$
4 K^{2}+(3+2 s) K-6 \sqrt{K+s} \sum_{k=1}^{K} \sqrt{k}-1+s \geq 0 .
$$

By the concavity of the square root we have

$$
\frac{\sqrt{k}+\sqrt{k+1}}{2} \leq \int_{k}^{k+1} \sqrt{t} \mathrm{~d} t
$$

Summing this from $k=1$ to $k=K-1$ we get

$$
\sum_{k=1}^{K} \sqrt{k} \leq \int_{1}^{K} \sqrt{t} \mathrm{~d} t+\frac{1+\sqrt{K}}{2}=\frac{2 K^{3 / 2}}{3}+\frac{K^{1 / 2}}{2}-\frac{1}{6} .
$$

This shows that

$$
\begin{aligned}
4 & K^{2}+(3+2 s) K-6 \sqrt{K+s} \sum_{k=1}^{K} \sqrt{k} \\
& \geq 4 K^{2}+(3+2 s) K-\sqrt{K(K+s)}(4 K+3)+\sqrt{K+s} \\
& =\frac{s K((4 s-12) K-9)}{4 K^{2}+(3+2 s) K+\sqrt{K(K+s)}(4 K+3)}+\sqrt{K+s} .
\end{aligned}
$$

In the quotient on the right side we estimate the numerator from below by $-3 s K(4 K+3)$ and the denominator from below by $4 K^{2}+3 K+K(4 K+3)=$ 
$2 K(4 K+3)$. Thus, the quotient is bounded from below by $-3 s / 2$, and since $K \geq 3$ we conclude that

$$
4 K^{2}+(3+2 s) K-6 \sqrt{K+s} \sum_{k=1}^{K} \sqrt{k}-1+s \geq \sqrt{K+s}-1-\frac{s}{2}>0 .
$$

This proves (14) and completes the proof of the lemma.

\subsection{PROOF OF THEOREM 1}

In this section, we are going to deduce Theorem 1 from Theorem 7. We define

$$
p(v):=-\frac{B}{2 \pi} \sum_{m=0}^{\infty}((2 m+1) B+v)_{-}
$$

for $v \in \mathbb{R}$. This is a concave, increasing and non-positive function. The key observation is that this $p$ is the Legendre transform of the function $-j$ from the previous subsection, that is,

$$
p(v)=\inf _{\rho \geq 0}(j(\rho)+v \rho) .
$$

This can be verified by elementary computations.

In order to prove Theorem 1 we apply Theorem 7 to get the estimate

$$
\operatorname{Tr}\left(H_{B}+V\right) \gamma \geq \int_{\mathbb{R}^{2}}\left(3 j\left(\rho_{\gamma}(x) / 3\right)+V(x) \rho_{\gamma}(x)\right) \mathrm{d} x
$$

for any $0 \leq \gamma \leq 1$. According to (15) this is bounded from below by $3 \int_{\mathbb{R}^{2}} p(V(x)) \mathrm{d} x$. For $\gamma$ equal to the projection corresponding to the negative spectrum of $H_{B}+V$ we obtain the assertion of Theorem 1 .

Remark 9. Similar arguments show that Theorem 7 can be deduced from Theorem 1. Indeed, since $j$ is convex it is its double Legendre transform. By (15) we obtain

$$
j(\rho)=\sup _{v \in \mathbb{R}}(p(v)-v \rho) .
$$

By the variational principle and Theorem 1 we can estimate for any $0 \leq \gamma \leq 1$ and any $V$

$$
\operatorname{Tr} H_{B} \gamma \geq-\operatorname{Tr}\left(H_{B}+V\right)_{-}-\int_{\mathbb{R}^{2}} V(x) \rho_{\gamma}(x) \mathrm{d} x \geq \int_{\mathbb{R}^{2}}\left(3 p(V(x))-V(x) \rho_{\gamma}(x)\right) \mathrm{d} x .
$$

Because of (16) we can choose the function $V$ such that the right side is equal to $3 \int_{\mathbb{R}^{2}} j\left(\rho_{\gamma}(x) / 3\right) \mathrm{d} x$, and this shows Theorem 7 . 


\section{Proof of Theorem 2}

\subsection{THE SPECTRUM OF $H_{B}+V$}

The explicit form of the eigenvalues of $H_{B}+\omega^{2}|x|^{2}$ was discovered in [5]. We include an alternative derivation of this result, which is also valid in the non-radial case.

LEMMA 10. For any $B>0$ and $\omega_{1}, \omega_{2}>0$ the operator $H_{B}+\omega_{1}^{2} x_{1}^{2}+\omega_{2}^{2} x_{2}^{2}$ has discrete spectrum and its eigenvalues, including multiplicities, are given by

$$
B\left(a_{+}\left(\frac{\omega_{1}}{B}, \frac{\omega_{2}}{B}\right)(2 k+1)+a_{-}\left(\frac{\omega_{1}}{B}, \frac{\omega_{2}}{B}\right)(2 l+1)\right), \quad k, l \in \mathbb{N}_{0},
$$

where

$$
a_{ \pm}\left(\sigma_{1}, \sigma_{2}\right)=\sqrt{\frac{1}{2}\left(1+\sigma_{1}^{2}+\sigma_{2}^{2} \pm \sqrt{\left.\left(1+\sigma_{1}^{2}+\sigma_{2}^{2}\right)^{2}-4 \sigma_{1}^{2} \sigma_{2}^{2}\right)}\right.} .
$$

Remark 11. It will be important for our analysis below that

$$
a_{-}(\sigma) a_{+}(\sigma)=\sigma_{1} \sigma_{2},
$$

which is easily checked.

Proof. By means of the gauge transform $\mathrm{e}^{-i B x_{1} x_{2} / 2}$ we see that $H_{B}+V$ is unitarily equivalent to the operator

$$
-\frac{\partial^{2}}{\partial x_{1}^{2}}+\left(-i \frac{\partial}{\partial x_{2}}-B x_{1}\right)^{2}+\omega_{1}^{2} x_{1}^{2}+\omega_{2}^{2} x_{2}^{2}
$$

which, in turn, by a partial Fourier transform with respect to $x_{2}$, is unitarily equivalent to

$$
-\frac{\partial^{2}}{\partial x_{1}^{2}}+\left(x_{2}-B x_{1}\right)^{2}+\omega_{1}^{2} x_{1}^{2}-\omega_{2}^{2} \frac{\partial^{2}}{\partial x_{2}^{2}} .
$$

After scaling $x_{2} \mapsto \omega_{2} x_{2}$ this becomes the non-radial harmonic oscillator $-\Delta+$ $x^{t} A x$ with the matrix

$$
A=\left(\begin{array}{cc}
B^{2}+\omega_{1}^{2} & -B \omega_{2} \\
-B \omega_{2} & \omega_{2}^{2}
\end{array}\right) .
$$

The eigenvalues of $A$ are $B^{2} a_{+}\left(\omega_{1} / B, \omega_{2} / B\right)^{2}$ and $B^{2} a_{-}\left(\omega_{1} / B, \omega_{2} / B\right)^{2}$. Using the eigenvectors of $A$ as basis in $\mathbb{R}^{2}$, we obtain a direct sum of two one-dimensional harmonic oscillators with frequencies $B a_{+}$and $B a_{-}$, respectively. This leads to the stated form of the eigenvalues. 
According to Lemma 10 and a simple computation, (5) with $\rho_{2}=1$ is equivalent to

$$
\begin{aligned}
& \sum_{k, l \geq 0}\left(\mu-B a_{+}\left(\frac{\omega_{1}}{B}, \frac{\omega_{2}}{B}\right)(2 k+1)-B a_{-}\left(\frac{\omega_{1}}{B}, \frac{\omega_{2}}{B}\right)(2 l+1)\right)_{+} \\
& \quad \leq \frac{B}{4 \omega_{1} \omega_{2}} \sum_{m \geq 0}(\mu-(2 m+1) B)_{+}^{2}
\end{aligned}
$$

with $a_{ \pm}$given by (17). Setting $\Lambda=\mu / B, \sigma_{j}=\omega_{j} / B$ and $a_{ \pm}=a_{ \pm}(\sigma)$ and substituting (18) we can rewrite the desired inequality as

$$
\sum_{k, l \geq 0}\left(\Lambda-a_{+}(2 k+1)-a_{-}(2 l+1)\right)_{+} \leq \frac{1}{4 a_{-} a_{+}} \sum_{m \geq 0}(\Lambda-(2 m+1))_{+}^{2}
$$

and this is what we shall prove.

\subsection{TWO INEQUALITIES FOR CONVEX FUNCTIONS}

For the proof of (19) we shall need

LEMMA 12. Let $\phi$ be a non-negative convex function on $(0, \infty)$ such that $\int_{0}^{\infty} \phi(t) d t$ exists. Then

$$
\sum_{k=0}^{\infty} \phi\left(k+\frac{1}{2}\right) \leq \int_{0}^{\infty} \phi(t) \mathrm{d} t
$$

Proof. Indeed, by the mean-value property of convex functions $\phi\left(k+\frac{1}{2}\right) \leq$ $\int_{k}^{k+1} \phi(t) \mathrm{d} t$ for each $k$. Now sum over $k$.

Remark 13. The proof also shows that $\sum_{k=0}^{K-1} \phi\left(k+\frac{1}{2}\right) \leq \int_{0}^{K} \phi(t) \mathrm{d} t$ for each integer $K$. This observation will be useful later.

The inequality from Lemma 12 is sufficient to prove a sharp Lieb-Thirring inequality in the non-magnetic case, but for the proof of our Theorem 2 we need a more subtle fact about convex functions. We note that by the previous lemma $h \sum_{k=0}^{\infty} \phi\left(h\left(k+\frac{1}{2}\right)\right) \leq \int_{0}^{\infty} \phi(t) \mathrm{d} t$ for any $h>0$. Moreover, $h \sum_{k=0}^{\infty} \phi\left(h\left(k+\frac{1}{2}\right)\right) \rightarrow$ $\int_{0}^{\infty} \phi(t) \mathrm{d} t$ as $h \rightarrow 0$ by the definition of the Riemann integral. The key for proving our sharp result is that, for a certain subclass of convex functions, this limit is approached monotonically. More precisely, one has

LEMMA 14. Let $\phi$ be a non-negative convex function on $(0, \infty)$ such that $\int_{0}^{\infty} \phi(t) \mathrm{d} t$ exists. Assume that $\phi$ is differentiable and that $\phi^{\prime}$ is concave. Then the sum 


$$
h \sum_{k=0}^{\infty} \phi\left(h\left(k+\frac{1}{2}\right)\right)
$$

is decreasing in the parameter $h>0$.

We emphasize that without assumptions on $\phi^{\prime}$ the inequality

$$
\sum_{k=0}^{\infty} \phi\left(k+\frac{1}{2}\right) \leq h \sum_{k=0}^{\infty} \phi\left(h\left(k+\frac{1}{2}\right)\right)
$$

is not true for all $h<1$. Indeed, take for instance $\phi(t)=(1-t)_{+}$and $h \geq 2 / 3$.

In the proof of this lemma we shall make use of the following well-known fact about convex functions: If $\psi$ is a non-negative convex function on $(0, \infty)$ such that $\int_{0}^{\infty} \psi(t) \mathrm{d} t$ exists, then $\psi(t)=\int_{0}^{\infty}(T-t)_{+} \mathrm{d} \mu(T)$ for some non-negative measure $\mu$. Indeed, it is known that the left-sided derivative $\partial_{-} \psi$ exists everywhere on $(0, \infty)$ and satisfies $\psi(b)-\psi(a)=\int_{a}^{b} \partial_{-} \psi(t) \mathrm{d} t$ for $0<a<b<\infty$. Moreover, $\partial_{-} \psi$ is increasing and left-continuous, and therefore there is a non-negative measure $\mu$ such that $\partial_{-} \psi(b)-\partial_{-} \psi(a)=\mu([a, b))$. Since $\lim _{t \rightarrow \infty} \psi(t)=\lim _{t \rightarrow \infty} \partial_{-} \psi(t)=0$, we have by Fubini's theorem

$$
\psi(t)=-\int_{t}^{\infty} \partial_{-} \psi(a) \mathrm{d} a=\int_{t}^{\infty}\left(\int \chi_{[a, \infty)}(T) \mathrm{d} \mu(T)\right) \mathrm{d} a=\int_{0}^{\infty}(T-t)_{+} \mathrm{d} \mu(T),
$$

as claimed.

Proof. According to the fact recalled above (applied to $\psi=-\phi^{\prime}$ ) we have $\phi(t)=\int_{0}^{\infty}(T-t)_{+}^{2} \mathrm{~d} \mu(T)$ for a non-negative measure $\mu$. Hence it suffices to prove the lemma for $\phi(t)=(T-t)_{+}^{2}$ with $T>0$. We have to prove that $\sum_{k=0}^{\infty}\left(\phi\left(h\left(k+\frac{1}{2}\right)\right)+h\left(k+\frac{1}{2}\right) \phi^{\prime}\left(h\left(k+\frac{1}{2}\right)\right) \leq 0\right.$, which for our $\phi$ reads

$$
\sum_{k=0}^{\infty}\left((S-2 k-1)_{+}^{2}-2(2 k+1)(S-2 k-1)_{+}\right) \leq 0
$$

with $S=2 T / h$. Choose $K \in \mathbb{N}_{0}$ such that $2 K-1 \leq S<2 K+1$. Then the left side above equals

$$
\begin{aligned}
& \sum_{k=0}^{K-1}\left((S-2 k-1)^{2}-2(2 k+1)(S-2 k-1)\right)=\sum_{k=0}^{K-1}\left(S^{2}-4 S(2 k+1)+3(2 k+1)^{2}\right) \\
& \quad=K\left(S^{2}-4 S K+(2 K-1)(2 K+1)\right)=K(S-2 K+1)(S-2 K-1) .
\end{aligned}
$$

This is clearly non-positive for $2 K-1 \leq S<2 K+1$, thus proving the claim. 


\subsection{PROOF OF THEOREM 2}

We have to prove (19). By Lemma 12 for any $k$

$$
\begin{aligned}
\sum_{l \geq 0}\left(\Lambda-a_{+}(2 k+1)-a_{-}(2 l+1)\right)_{+} & \leq \int_{0}^{\infty}\left(\Lambda-a_{+}(2 k+1)-2 a_{-} t\right)_{+} \mathrm{d} t \\
& =\frac{1}{4 a_{-}}\left(\Lambda-a_{+}(2 k+1)\right)_{+}^{2} .
\end{aligned}
$$

A simple computation shows that $a_{+}=a_{+}(\sigma) \geq 1$, and hence by Lemma 14

$$
a_{+} \sum_{k \geq 0}\left(\Lambda-a_{+}(2 k+1)\right)_{+}^{2} \leq \sum_{k \geq 0}(\Lambda-(2 k+1))_{+}^{2} .
$$

The previous two inequalities imply the desired (19).

\subsection{PROOF OF PROPOSITION 3}

Given $0 \leq \gamma<1$, we want to find $\omega_{1}=\omega_{2}$ and $B$ such that the reverse inequality (7) holds. We may assume $\gamma>0$ in the following. (The case $\gamma=0$ can be treated similarly, or one may use the argument of Aizenman and Lieb mentioned in the introduction to conclude that a counterexample for $\gamma=\gamma_{0}$ implies one for all $\gamma<\gamma_{0}$.)

By the same computation that lead to (19) we see that (7) can be written as:

$$
\sum_{k, l \geq 0}\left(\Lambda-a_{+}(2 k+1)-a_{-}(2 l+1)\right)_{+}^{\gamma}>\frac{1}{2(\gamma+1) a_{-} a_{+}} \sum_{m \geq 0}(\Lambda-(2 m+1))_{+}^{\gamma+1}
$$

with $\Lambda=\mu / B, \sigma_{j}=\omega_{j} / B$ and $a_{ \pm}=a_{ \pm}(\sigma)$. We will let $\omega_{1}=\omega_{2}$ and use the notation $t=\sigma^{2}$. One can show that $a_{+}=1+t+O\left(t^{2}\right)$ and $a_{-}=t+O\left(t^{2}\right)$ as $t \rightarrow 0+$. We now choose $\Lambda=3$ and recall that $a_{+}=a_{+}(\sigma) \geq 1$. This gives us the inequality

$$
2(\gamma+1) a_{-} a_{+} \sum_{l \geq 0}\left(3-a_{+}-a_{-}(2 l+1)\right)_{+}^{\gamma}-2^{\gamma+1}>0,
$$

which may be written as

$$
(\gamma+1) a_{-}^{\gamma+1} a_{+} \sum_{l \geq 0}(x-l)_{+}^{\gamma}-1>0
$$

with $x=\left(3-a_{+}-a_{-}\right) /\left(2 a_{-}\right)$. Since $x=t^{-1}(1+O(t))$ as $t \rightarrow 0+$, we may choose $\sigma$ so that $x$ is an integer. In this case we may use the concavity of $y^{\gamma}$ and Remark 13 to bound

$$
\sum_{l \geq 0}(x-l)_{+}^{\gamma}=\sum_{l=1}^{x} l^{\gamma} \geq \int_{1 / 2}^{x+1 / 2} t^{\gamma} \mathrm{d} t=\frac{1}{\gamma+1}\left((x+1 / 2)^{\gamma+1}-(1 / 2)^{\gamma+1}\right) .
$$


This shows that

$$
\begin{aligned}
(\gamma+1) a_{-}^{\gamma+1} a_{+} \sum_{l \geq 0}(x-l)_{+}^{\gamma} & \geq a_{+}\left(\left(a_{-} x+a_{-} / 2\right)^{\gamma+1}-\left(a_{-} / 2\right)^{\gamma+1}\right) \\
& =a_{+}\left(\left(\left(3-a_{+}\right) / 2\right)^{\gamma+1}-\left(a_{-} / 2\right)^{\gamma+1}\right) \\
& =\left(1+t+O\left(t^{2}\right)\right)\left(1-t / 2+O\left(t^{2}\right)\right)^{\gamma+1}+O\left(t^{\gamma+1}\right) \\
& =1+\frac{1-\gamma}{2} t+O\left(t^{\gamma+1}\right) .
\end{aligned}
$$

Since this is strictly larger than 1 for sufficiently small $t$, we have proved our claim.

\section{Acknowledgements}

R. Olofsson acknowledges support through the Swedish Research Council.

\section{References}

1. Aizenman, M., Lieb, E.H.: On semiclassical bounds for eigenvalues of Schrödinger operators. Phys. Lett. A 66(6), 427-429 (1978)

2. de la Bretèche, R.: Preuve de la conjecture de Lieb-Thirring dans le cas des potentiels quadratiques strictement convexes. (French) Ann. Inst. H. Poincaré Phys. Théor. 70(4), 369-380 (1999)

3. Dolbeault, J., Laptev, A., Loss, M.: Lieb-Thirring inequalities with improved constants. J. Eur. Math. Soc. 10, 1121-1126 (2008)

4. Erdős, L., Loss, M., Vougalter, V.: Diamagnetic behavior of sums of Dirichlet eigenvalues. Ann. Inst. Fourier 50(3), 891-907 (2000)

5. Fock, V.: Bemerkung zur Quantelung des harmonischen Oszillators im Magnetfeld. (German) Zeitschrift Physik 47, 446-448 (1928)

6. Frank, R.L.: Remarks on eigenvalue estimates and semigroup domination. In: Briet, P., et al. (eds.) Spectral and Scattering Theory for Quantum Magnetic Systems. Contemp. Math., vol. 500, pp. 63-86. Amer. Math. Soc., Providence (2009)

7. Frank, R.L., Loss, M., Weidl, T.: Pólya's conjecture in the presence of a constant magnetic field. J. Eur. Math. Soc. (JEMS) 11(6), 1365-1383 (2009)

8. Helffer, B., Robert, D.: Riesz means of bounded states and semi-classical limit connected with a Lieb-Thirring conjecture. II. Ann. Inst. H. Poincaré Phys. Théor. 53(2), 139-147 (1990)

9. Hundertmark, D., Laptev, A., Weidl, T.: New bounds on the Lieb-Thirring constants. Invent. Math. 40, 693-704 (2000)

10. Laptev, A.: Dirichlet and Neumann eigenvalue problems on domains in Euclidean spaces. J. Funct. Anal. 151(2), 531-545 (1997)

11. Laptev, A.: On the Lieb-Thirring conjecture for a class of potentials. In: The Maz'ya Anniversary Collection, vol. 2 (Rostock, 1998). Oper. Theory Adv. Appl., vol. 110, pp. 227-234. Birkhäuser, Basel (1999)

12. Laptev, A., Weidl, T.: Sharp Lieb-Thirring inequalities in high dimensions. Acta Math. 184(1), 87-111 (2000)

13. Lieb, E.H., Solovej, J.P., Yngvason, J.: Asymptotics of heavy atoms in high magnetic fields II. Semiclassical regions. Commun. Math. Phys. 161(1), 77-124 (1994) 
14. Lieb, E.H., Solovej, J.P., Yngvason, J.: Ground states of large quantum dots in magnetic fields. Phys. Rev. B 51(16), 10646-10665 (1995)

15. Lieb, E.H., Thirring, W.: Inequalities for the moments of the eigenvalues of the Schrödinger Hamiltonian and their relation to Sobolev inequalities. In: Studies in Mathematical Physics, pp. 269-303. Princeton University Press, Princeton (1976)

16. Rumin, M.: Balanced distribution-energy inequalities and related entropy bounds. Preprint (2010). arXiv:1008.1674 